\title{
Subjective recalibration of advisors' probability estimates
}

\author{
YARON Shlomi AND ThOMAS S. WALlSTEN \\ University of Maryland, College Park, Maryland
}

\begin{abstract}
Are decision makers sensitive to the statistical properties (i.e., calibration) of probability estimates that they receive from advisors? After specifying the ideal use of such estimates, we derive the roughly ideal forecast consumer (RIFC) and generalize it to account for how humans might use the estimates. We report an experiment in which participants first experienced various advisors by seeing their probability estimates and the associated outcomes and then provided confidence judgments in the presence of the advisors' estimates. The generalized model described the data well and showed that the participants were appropriately sensitive to the statistical properties of the advisors. Models of the individuals were better calibrated than the participants themselves, but still inferior to the RIFC. A detailed description of our model-testing procedure can be found in an appendix to the article.
\end{abstract}

Decision makers (DMs) often rely on probabilistic estimates from external sources to guide their behavior. Examples include investment decisions made on the basis of market forecasts and military and/or diplomatic actions guided by intelligence forecasts. This research explores the DMs' use of the estimates as a function of their quality.

The report is organized as follows: First, we review pertinent conceptual and methodological aspects of measuring the quality of the estimates. Then, we briefly summarize the evidence regarding how DMs use estimates provided by external sources. Finally, we develop a cognitively plausible model for using the estimates, which we call the roughly ideal forecast consumer (RIFC) because it yields close to optimal performance, and use it in an experiment as a baseline against which to assess human performance. ${ }^{1}$ We conclude with theoretical implications of the results.

\section{Calibration of Probabilities}

The correspondence between estimates and actual events is the topic of an extensive literature (e.g., Wallsten, Budescu, Erev, \& Diederich, 1997). For our purposes, a discussion of calibration suffices. An estimate is well calibrated if it matches the relative frequency of the events conditional on the estimate. To illustrate, a precipitation forecast of $60 \%$ is well calibrated if rain is observed within the forecast area on 60 of 100 days on which the forecast is used.

We distinguish between the estimate and the observed relative frequency conditional on the estimate by referring to the former as the subjective probability (SP) and the latter as the objective probability (OP). The calibration measure is typically used to characterize the quality of a distribution of estimates, each associated with a dif- ferent event. In this context, calibration is a measure of the extremity of the OPs relative to the extremity of the corresponding SPs (see, e.g., Erev, Wallsten, \& Budescu, 1994; Wallsten et al., 1997; Yates, 1982, 1990). According to this definition, a set of SPs is well calibrated if (in the long run) OP equals SP for all SP; it is overconfident when SP is more extreme than OP; and it is underconfident when SP is less extreme than OP. The cases are depicted by three advisors' calibration curves in the left panel of Figure 1, which graphs OP (times 100) as a function of advisor estimate, SP (times 100). The main diagonal represents a well-calibrated advisor, for whom OP $=$ SP for all values of SP. The curve that begins below the diagonal, crosses it at the middle, and continues above the diagonal represents an underconfident advisor, in that events occur (or fail to occur) with more extreme probabilities than he or she estimates. The remaining curve represents an overconfident advisor, in that events occur (or fail to occur) with less extreme probabilities than he or she estimates.

Ideal use of an advisor's estimate requires treating the advisor's SP, $p$, not as a probability, but simply as a datum that signals the event $\mathrm{E}$ with some OP (cf. Clemen \& Murphy, 1990; French, 1986; Winkler, 1990). In other words, the ideal use of the estimate involves rescaling (i.e., recalibrating) the SPs to the OPs they actually represent. Our primary research question is whether - and if so, howhuman decision makers rescale such estimates.

Previous research tentatively suggests that participants are affected by the calibration of their advisors, but the work is mute regarding how participants actually rescale the estimates. Specifically, Price and Stone (2004) presented participants with two advisors who made a series of forecasts. Participants also saw the outcomes, and thus 

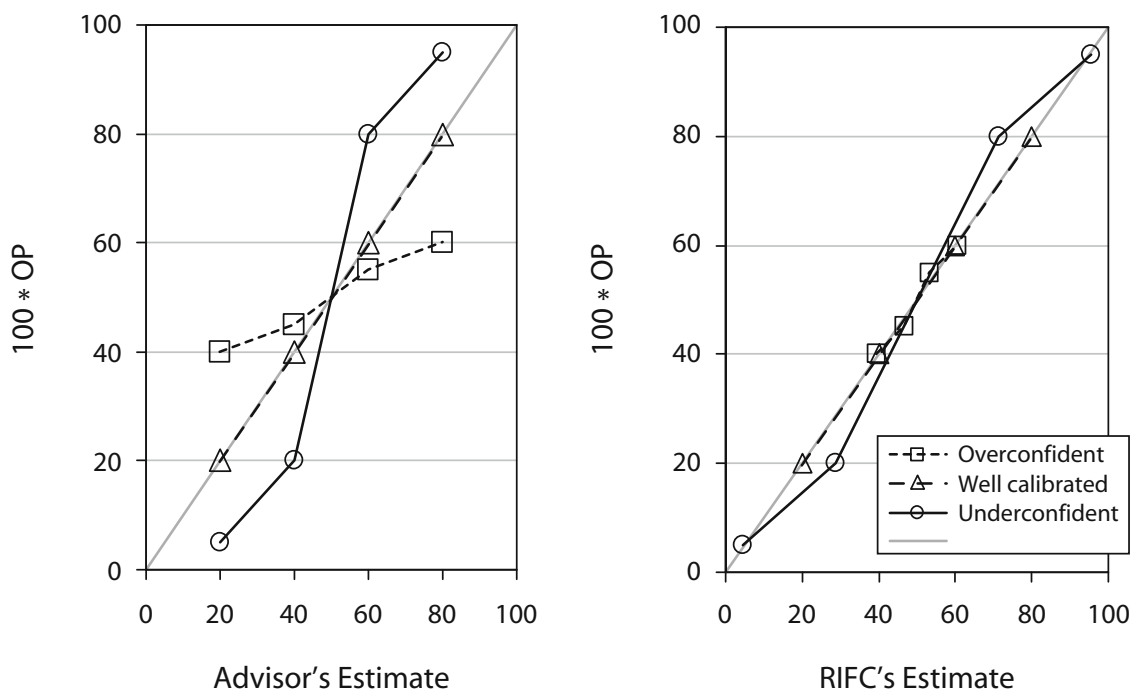

Figure 1. Calibration curves of the illustrative advisors (left panel) and the roughly ideal forecast consumer (right panel).

could learn about the advisors' calibration. The series of forecast-outcome pairings were devised so that the two advisors were differentially calibrated. At the end of this training phase, participants were asked which advisor they would prefer to hire. The primary result was that most decision makers preferred to hire the overconfident rather than the well-calibrated advisor (for related work, see Keren \& Teigen, 2001; Yates, Price, Lee, \& Ramirez, 1996; see also Budescu \& Yu, 2007). Because participants were only asked for their preference, it is not possible to determine how they would have used (i.e., rescaled) the advisors' estimates in arriving at their own judgments.

To summarize: Other than the studies mentioned above, no research to our knowledge has looked at whether-and if so, how-decision makers use advisors' SP estimates in forming their own judgments. ${ }^{2}$ We next consider a cognitively plausible, approximately optimal model for such information use, which we call the RIFC.

\section{The Roughly Ideal Forecast Consumer}

Ideal forecast consumers (IFCs) must learn an advisor's distribution of estimates when event E occurs and when it does not - that is, $P(p \mid \mathrm{E})$ and $P\left(p \mid \mathrm{E}^{c}\right)$, respectively, for all values of $p$. Using Bayes's rule, the IFC combines its prior probability with the experienced conditional distribution of events to obtain a posterior probability $t=P(\mathrm{E} \mid p)$ :

$$
t=\frac{P(\mathrm{E} \cap p)}{P(p)}=\frac{P(p \mid \mathrm{E}) P(\mathrm{E})}{P(p \mid \mathrm{E}) P(\mathrm{E})+P\left(p \mid \mathrm{E}^{c}\right) P\left(\mathrm{E}^{c}\right)}
$$

The IFC recalibrates on a relative frequency basis that entails substantial cognitive resources (e.g., working memory capacity) and sufficient experience with the advisor to form a reliable estimate of $t$ for each value of $p$. And the more values of $p$ one has to keep track of, the greater the required cognitive resources. For example, if the advisor provides SP estimates of 0 to 1 in units of .10 (or, equivalently, confidence estimates from $0 \%$ to $100 \%$ in units of $10 \%$ ), the IFC would need to keep track of and update 11 values of $t$.

We believe that the dual requirement of extensive experience and ample cognitive resources precludes the IFC as a plausible model of the unaided human forecast consumer. Thus, we create a more plausible model of the unaided human forecast consumer by modifying the IFC into a roughly ideal forecast consumer (RIFC).

To simplify the exposition of the RIFC, we begin with a function relating $p$ to $t$ that has convenient properties (Karmarkar, 1978):

$$
t=\frac{p^{s}}{p^{s}+(1-p)^{s}} .
$$

It often is useful to express the function in its odds or logodds form:

$$
\frac{t}{1-t}=\left(\frac{p}{1-p}\right)^{s}, \text { or } \log \left(\frac{t}{1-t}\right)=s \log \left(\frac{p}{1-p}\right) \text {. }
$$

The function specifies that any particular value of $p$ can be transformed into a corresponding $t$ via a specific value of $s$. It is unlikely, however, that a single value of $s$ will allow a perfect transformation of the entire set of $p$ s into the corresponding set of $t \mathrm{~s}$. Therefore, the RIFC updates a single value $s$ that brings the entire set of $p$ s into close alignment with a set of approximated objective probabilities. Building on the log-odds form of Equation 2, the RIFC estimates a value of $s$ that minimizes $d$ in the following equation:

$$
d=\sum\left[\log \left(\frac{t}{1-t}\right)-s \log \left(\frac{p}{1-p}\right)\right]^{2} .
$$

A function such as Equation 3 allows the RIFC to use observations across all values of $p$ for the purpose of approximating values of $t$ and thus can yield good levels of recalibration with far less experience than is otherwise required. The primary disadvantage is that it may not adapt 
well to some calibration curves. We do not imagine that human forecast consumers estimate their scaling factor $s$ in this fashion and offer a suggestion in the Discussion section about how they might do so.

The right-hand panel of Figure 1 illustrates the RIFC's recalibration of the overconfident, well-calibrated, and underconfident advisors shown in the left-hand panel. The scaling factors, $s$, estimated by minimizing Equation 3 are $0.31,1.00$, and 2.23 , respectively.

A comparison between the two panels of Figure 1 shows that the RIFC's estimates, conditional on the estimates of the overconfident and underconfident advisor, are closer to proper calibration (i.e., the main diagonal) than are the advisor's estimates. Although better calibrated than the advisor's estimates, the rescaled estimates are not always precisely on the diagonal. Thus, although Equations 2 and 3 are quite flexible, the RIFC cannot use them to accommodate all possible calibration curve patterns. Because the optimality of the RIFC depends on the correspondence between the advisor's calibration curve and the recalibration function, the RIFC is optimal to greater or lesser degrees.

\section{The Human Forecast Consumer}

We believe that, like the RIFC, the human forecast consumer does not learn discrete values of $t$ (the objective probabilities), but rather seeks a functional relationship between $p$ s and $t$ s. Formally, we modify the odds version of Equation 2 to account for the recalibration of the human forecast consumer,

$$
\frac{\bar{r}}{1-\bar{r}}=\left(\frac{p}{1-p}\right)^{s} .
$$

Now, the left-hand side of Equation 4 is the DM's mean estimate $\bar{r}$ over multiple occurrences of the advisor's judgment $p$. The use of $\bar{r}$ instead of $r$ acknowledges that individual trial-by-trial DM responses will vary around a central value for each value of $p$. The value of $s$ in Equation 4 yields a measure of the human forecast consumer's recalibration (i.e., scaling) of the advisor's judgments.

The experiment that follows addresses three questions: (1) Does the model implied by Equation 4 reasonably describe how human forecast consumers rescale the estimates? (2) If the answer is affirmative, how do the participants' scale factors compare with the optimal RIFC values? (3) Finally, how well do participants and the participants' model recalibrate relative to the RIFC?

In our procedure, adapted from Price and Stone (2004), participants learned about and then relied on a number of differentially calibrated advisors. Each block of trials corresponded to one advisor and involved a learning phase followed by a test phase. The learning phase provided participants with experience that allowed them to form a subjective representation of an advisor's calibration. Participants gained this experience by observing a set of statements. For each statement, they saw the advisor's estimate that it was true and feedback as to whether in fact it was true. Following the learning phase was a test phase designed to examine how participants used the advisor's estimate, given the immediately preceding experience. Participants observed a new set of statements, each accompanied by the advisor's estimate. Given the statement and the advisor's estimate, participants were required to provide their own estimate. There was no trial-by-trial feedback in the test phase.

\section{METHOD}

\section{Participants}

Thirty undergraduates at the University of Maryland participated in the experiment for course credit.

\section{Stimuli and Procedure}

The stimuli were 672 statements about the relative unemployment rates ${ }^{3}$ in pairs of U.S. states, of the form, "X had a LOWER unemployment rate than Y." The procedure started with a brief overview of the task. The participants were told that they would form an impression about an advisor by observing him make a set of judgments and receiving feedback about the correctness of those judgments. Participants were also told that in a subsequent part of the experiment they would receive the advisor's judgments and then be required to make their own. We provided no prescription for how the advisor's judgments should be used and did not mention the notion of calibration

Participants saw a screenshot of a training trial and an explanation of the information contained therein prior to the start of training. Similar information was provided before the first testing trial.

Three advisors were presented in one of three orders that were counterbalanced across participants. The three advisors differed in that one was well calibrated, another was underconfident, and one was overconfident. There were 160 trials in a training block and 64 trials in a testing block for each advisor.

The learning trials provided sufficient information for the participant to learn about each advisor's level of calibration. For each trial, a statement appeared on the screen along with the picture and name of the advisor, and the advisor's estimate that the statement was true. All target statements were of the form "X had a LOWER unemployment rate than Y" (e.g., Georgia had a LOWER unemployment rate than Iowa). When the target statement was correct, the feedback "X had a LOWER unemployment rate than Y" appeared in blue font. When the target statement was wrong, the feedback " $X$ had a HIGHER unemployment rate than Y" appeared in red font.

The test trials immediately followed the learning trials for each advisor. On each trial, participants saw a statement, received an advisor's estimate that it was true, and then gave their own estimate. Testing trials differed from the training trials in two ways: (1) There was no feedback (the correct statement was not given), and (2) participants provided their estimates with a slider anchored Definitely false on the left end and Definitely true on the right end, but otherwise uncalibrated. Responses were recorded as 0 to 100 , as a function of the relative position in which the participant set the slider.

The advisor's estimate on each training and testing trial was selected from the following values: $20 \%, 40 \%, 60 \%$, and $80 \%$. Each estimate was selected 40 and 16 times on each advisor's training and testing blocks, respectively. Given percentage estimates of $20 \%$, $40 \%, 60 \%$, and $80 \%$, the proportion of true statements that were sampled for the overconfident advisor were $.35, .45, .55$, and .65 ; for the calibrated advisor, $.20, .40, .60$, and .80 ; and for the underconfident advisor, $.05, .15, .85$, and .95 . The calibration curves corresponding to these distributions are presented in Figure 2.

\section{RESULTS}

The purpose of the analyses is to summarize the relationship between the participants' judgments as a function 


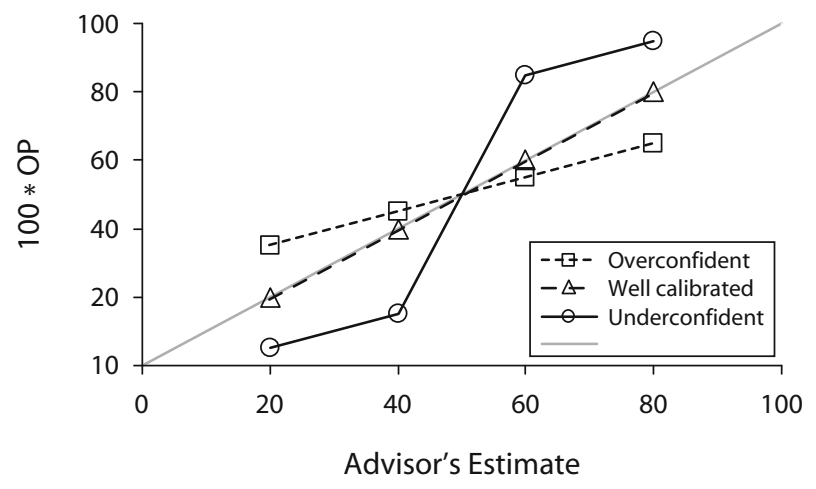

Figure 2. The advisors' calibration curves.

of the advisor's estimate and the advisor's calibration. We believe that the participants were unfamiliar with the task domain (unemployment rates) and thus expect the advisor's estimate and the statistical properties (i.e., the calibration) of the advisors' estimates to be the primary influences on the participants' judgments.

Prior to all analyses, we converted responses to log-odds by dividing the estimates by 100 to yield probabilities, $r$, and applying the transformation $r^{\prime}=\ln [r /(1-r)]$. We transformed the responses back to the $0-100$ scale for purposes of presentation. Because $r^{\prime}$ is undefined when $r$ equals 0 or 1 , we substituted .005 and .995 , respectively, in those cases (for a similar approach, see Dhami \& Wallsten, 2005).

The points shown as asterisks in Figure 3 show the mean judgments as a function of the advisor's estimates, with a separate panel for each advisor. Judgments were most extreme given the underconfident advisor and least extreme given the overconfident advisor.

\section{Model-Based Analyses}

Question 1 is whether the model embodied in Equation 4 reasonably describes the mean judgments. To address the issue, we first write the model in a manner that applies to individual trials, which include variability, by assuming a multiplicative error term $\varepsilon$. Thus, using $j$ and $k$ to denote the advisor and the trial, respectively, and assuming that the recalibration factor $s$ and the variance of the error term $\varepsilon$ vary with the judge, the model can be expressed as

$$
\frac{r_{j k}}{1-r_{j k}}=\left(\frac{p_{j k}}{1-p_{j k}}\right)^{s_{j}} \varepsilon_{j k} .
$$

Taking logs yields the more convenient form,

$$
\ln \left(\frac{r_{j k}}{1-r_{j k}}\right)=s_{j} \ln \left(\frac{p_{j k}}{1-p_{j k}}\right)+\ln \left(\varepsilon_{j k}\right),
$$

or continuing with the notation introduced above, letting $r^{\prime}=\ln [r /(1-r)], p^{\prime}=\ln [p /(1-p)]$, and $\varepsilon^{\prime}=\ln (\varepsilon)$,

$$
r_{j k}^{\prime}=s_{j} p_{j k}^{\prime}+\varepsilon_{j k}^{\prime} \text {. }
$$

We used maximum likelihood procedures to estimate the model separately for each participant, assuming (1) the error term $\varepsilon^{\prime}$ is identically normally distributed with mean 0 and standard deviation $\sigma_{j}$ for each advisor $j$, (2) trials are independent, and (3) the empirically derived pooled standard deviations of $r^{\prime}$ provide unbiased estimates of the $\sigma_{j}$. The model, thus, has three free parameters per participant: $s_{1}, s_{2}$, and $s_{3}$. To test whether the participants distinguished
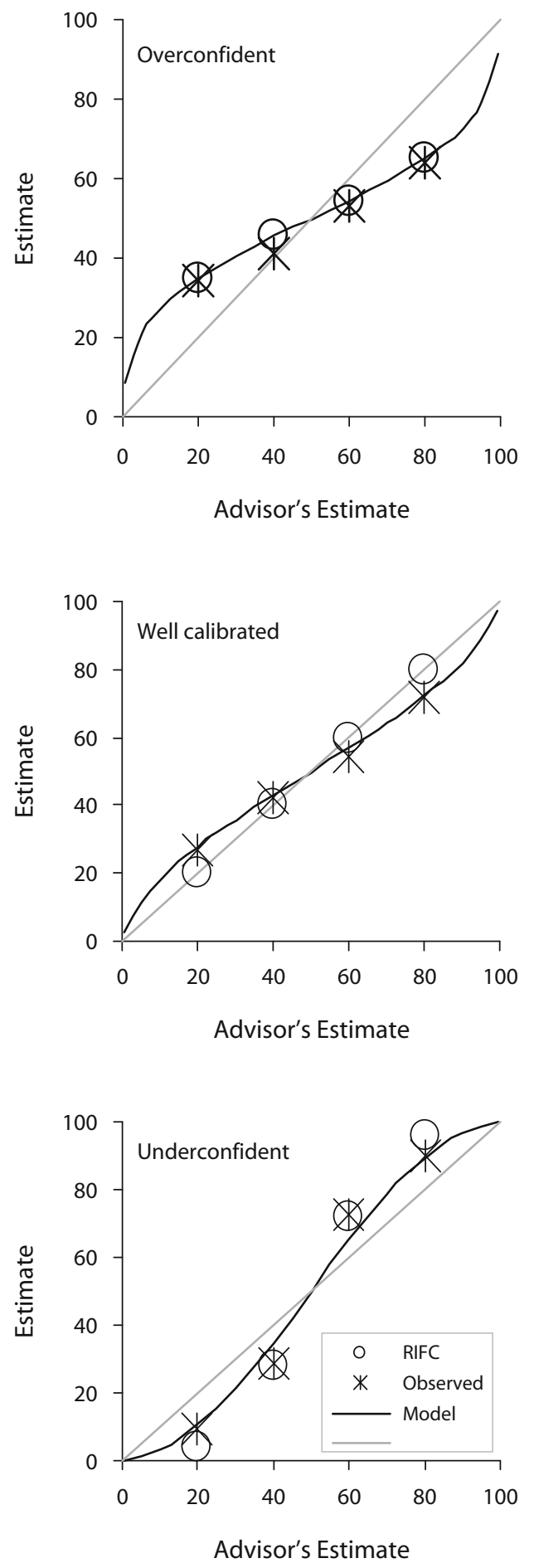

Figure 3. Roughly ideal forecast consumer (RIFC), observed, and model judgments as a function of the advisor's estimates. 
among the advisors, we used maximum likelihood ratio tests to compare the unequal-scale-factor model to a constrained model in which $s_{1}=s_{2}=s_{3}$. The resulting $G^{2}$ statistic is asymptotically chi-square distributed with $d f=2$. Additional details about the maximum likelihood ratio tests and $G^{2}$ statistic are described in the Appendix.

The unequal-scale model provided a better fit to the data (at $\alpha=.05$ ) than did the equal-scale model for 26 out of 30 participants. Thus, we can reject the equal-scale model as a likely description of the participants' estimates, and conclude that participants were sensitive to the calibration of the advisors' estimates. The estimated scaled factors accorded to the overconfident, well-calibrated, and underconfident advisor were $0.45,0.69$, and 1.55 , respectively.

The mean model-derived judgments, shown as the curves in Figure 3, compare well with the mean data values. Comparing model and data responses at the level of individual participants, the average squared linear correlations $\left(r^{2}\right)$ between the two were $.72, .75$, and .90 for the overconfident, well-calibrated, and underconfident ${ }^{4}$ advisors, respectively. On this basis, it appears that the model provides an excellent description of the data at the level of individual participants.

\section{Comparing Empirical and RIFC (Optimal) Scale Factors}

Question 2 asks how the empirically estimated scale factors compare with the RIFC's (optimal) values. We quantified this relationship by computing, for each participant, the correlation between the optimal RIFC values and the participant's estimates. The average squared linear correlation $\left(r^{2}\right)$ between the participants' estimates and the RIFC estimates is .73, indicating a strong relationship between the two. The open points in Figure 3 show the RIFC's probability estimates obtained by using the optimal scale values. They correspond very closely to the mean empirical judgments.

That the empirical scale factors are on average less extreme than the optimal (RIFC's) values can be seen in

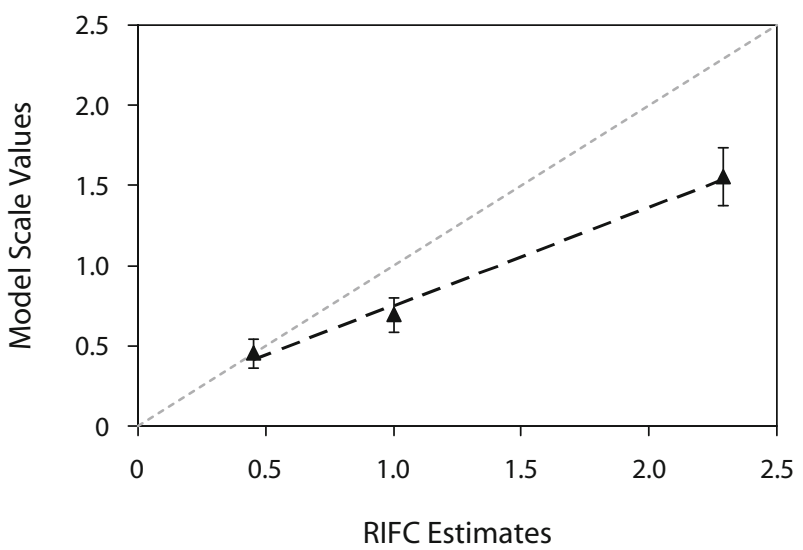

Figure 4. Model scale values as a function of roughly ideal forecast consumer (RIFC) scale values. Moving from left to right, the data points correspond to the overconfident, well-calibrated, and underconfident advisors. Error bars correspond to $1 S E$.
Figure 4, which plots the former as a function of the latter. Linearly regressing the model-based scale factors for each participant on the RIFC's scale factors yields slopes that are on average $(M=.61, S E=.10)$ significantly less than one $[t(29)=3.86, p<.001]$. However, the intercepts $(M=.14, S E=.11)$ are not different from zero $[t(29)=$ $1.23, p=.21]$.

\section{Quality of Recalibration}

Question 3 asks how well the participants and the participants' models recalibrate relative to the RIFC. To answer these questions, we computed a recalibration score (RS) as the mean absolute deviation (MAD) between the log-odds of the objective probabilities conditional on the advisors' estimates and the log-odds of a set of recalibrated estimates (from the participant, the model of the participant, or the RIFC). We then averaged the RS values across the three advisors, yielding one RS for each set of estimates (the participant, the model of the participant, and the RIFC). Note that lower values of RS imply better performance.

The participants' RS ( $M=.77, S E=.05)$ was significantly greater than the RS of their models $(M=.69, S E=$ $.05)[t(29)=2.35, p<.01]$, which was significantly greater than the RIFC's RS $(M=.12)[t(29)=13.27, p<.01]$. The RS analysis shows that the RIFC better approximated the advisor's OP than did either the participants or the participants' models. Note that this comparison could have turned out differently if the mechanism that humans use to recalibrate was more effective (flexible) than the RIFC.

\section{DISCUSSION}

We began with three questions. The answer to the first, does the model implied by Equation 4 reasonably describe how human forecast consumers rescale advisors' estimates, is affirmative. The answer to the second, how do the estimated scale factors compare with the optimal RIFC values, is that the best-fitting scale factors are less extreme than the optimal values, probably as a result of random trial-by-trial variability inherent in the responses.

The reply to the third, how well do participants and their models recalibrate relative to the RIFC, is that the RIFC's recalibration is the closest to ideal, the models of the participants are next closest, and the participants themselves are least well calibrated. But it should not be overlooked that the participants' performance nevertheless is still very good. The fact that the models of the judges outperform the judges has been found in other judgment tasks, such as diagnosis on the basis of probabilistic cues (e.g., Goldberg, 1970), and most likely is a consequence of the models' averaging out the effects of random error.

A remaining open - and very important-question concerns the cognitive processes that underlie this subjective rescaling of advisors' estimates. One possibility is that it is due to a set of direct associations between each of the estimates in an advisor's lexicon (the advisor's SPs) and consequent OP (cf. McDaniel \& Busemeyer, 2005). However, as noted in the introduction, this strategy entails 
the capacity to store and regularly update multiple streams of experience - that is, to store and update distinct values of $t$ for each $p$ and for each advisor. The forecast consumer's cognitive strain would be substantial, and therefore we doubt that this is the operative mechanism.

Rather than storing and retrieving the OP associated with individual estimates, it is more likely that decision makers recalibrate advisors' estimates by forming and using a representation that integrates the experiences accrued across different estimates (cf. rule learning, McDaniel \& Busemeyer, 2005). We know that humans can learn and use functional relationships between predictor variable(s) and a criterion variable (Brehmer, 1974; Kalish, Lewandowsky, \& Kruschke, 2004; Kelley \& Busemeyer, 2008; McDaniel \& Busemeyer, 2005), but the criterion variable $(t)$ associated with each predictor $(p)$ in this case is inferred from experience, not observed directly, so the learning process very likely is different in this case than in others.

Participants received 160 training trials with each advisor (during which we collected no data) prior to the 64 test trials, which yielded the data analyzed here. Thus, we had no opportunity to observe or test hypotheses about the learning process. Assuming the participants implicitly adopted a calibration function that is reasonably represented by Equation 4, we may hypothesize that instead of keeping track of multiple estimates of $t$, they updated the scale factor $s$ following feedback on each training trial. Models and empirical studies of how they accomplish this are the subject of current research and will be described in subsequent reports.

\section{AUTHOR NOTE}

We thank David Budescu for comments on a previous version of this article. Portions of this work were presented in 2006 at the Workshop on Advice and Trust in Decision Making held at University College London; in 2006 at the annual meeting of the Society for Judgment and Decision Making in Houston, Texas; in 2007 at a workshop titled Meteorology Meets Social Science: Risk, Forecast and Decision, held at the University of Exeter; and in 2007 at the annual meeting of the Psychonomic Society in Long Beach, California. Correspondence concerning this article should be addressed to Y. Shlomi, Department of Psychology, University of Maryland, College Park, MD 20742-4411 (e-mail: yshlomi@psyc.umd.edu).

\section{REFERENCES}

BReHMER, B. (1974). Hypotheses about relations between scaled variables in the learning of probabilistic inference tasks. Organizational Behavior \& Human Performance, 11, 1-27.

Budescu, D. V., \& Yu, H.-T. (2007). Aggregation of opinion based on correlated cues and advisors. Journal of Behavioral Decision Making, 20, 153-177.

Clemen, R. T., \& Murphy, A. H. (1990). The expected value of frequency calibration. Organizational Behavior \& Human Decision Processes, 46, 102-117.

Dhami, M. K., \& Wallsten, T. S. (2005). Interpersonal comparison of subjective probabilities: Toward translating linguistic probabilities. Memory \& Cognition, 33, 1057-1068.

Erev, I., Wallsten, T. S., \& Budescu, D. V. (1994). Simultaneous over- and underconfidence: The role of error in judgment processes. Psychological Review, 101, 519-527.

FrenCH, S. (1986). Calibration and the expert problem. Management Science, 32, 315-321.

GoldBERG, L. R. (1970). Man versus model of man: A rationale plus some evidence for a method of improving on clinical inferences. Psychological Bulletin, 73, 422-432.

Kalish, M. L., Lewandowsky, S., \& Kruschke, J. K. (2004). Population of linear experts: Knowledge partitioning and function learning. Psychological Review, 111, 1072-1099.

KARMARKAR, U. S. (1978). Subjectively weighted utility: A descriptive extension of the expected utility model. Organizational Behavior \& Human Performance, 21, 61-72.

Kelley, H., \& BusemeYer, J. (2008). Comparison of models for learning how to dynamically integrate multiple cues to forecast continuous criteria. Journal of Mathematical Psychology, 52, 218-240.

Keren, G., \& Teigen, K. H. (2001). Why is $p=.90$ better than $p=.70$ ? Preference for definitive predictions by lay consumers of probability judgments. Psychonomic Bulletin \& Review, 8, 191-202.

MCDANiel, M. A., \& BusemeYer, J. R. (2005). The conceptual basis of function learning and extrapolation: Comparison of rule-based and associative-based models. Psychonomic Bulletin \& Review, 12, 24-42.

Price, P. C., \& Stone, E. R. (2004). Intuitive evaluation of likelihood judgment producers: Evidence for a confidence heuristic. Journal of Behavioral Decision Making, 17, 39-57.

RiefER, D. M., \& BATChelder, W. H. (1988). Multinomial modeling and the measurement of cognitive processes. Psychological Review, $\mathbf{9 5}, 318-339$

Wallsten, T. S., Bender, R. H., \& LI, Y. (1999). Dissociating judgment from response processes in statement verification: The effects of experience on each component. Journal of Experimental Psychology: Learning, Memory, \& Cognition, 25, 96-115.

Wallsten, T. S., Budescu, D. V., Erev, I., \& Diederich, A. (1997). Evaluating and combining subjective probability estimates. Journal of Behavioral Decision Making, 10, 243-268.

WINKLER, R. L. (1990). Representing and communicating uncertainty. Statistical Science, 5, 26-30.

YATES, J. F. (1982). External correspondence: Decompositions of the mean probability score. Organizational Behavior \& Human Performance, 30, 132-156.

YATES, J. F. (1990). Judgment and decision making. Englewood Cliffs, NJ: Prentice Hall.

Yates, J. F., Price, P. C., Lee, J.-W., \& RamireZ, J. (1996). Good probabilistic forecasts: The consumer's perspective. International Journal of Forecasting, 12, 41-56.

\section{NOTES}

1. We use "forecast consumers" and "decision makers" interchangeably. 2. Budescu and Yu (2007) investigated how DMs used SP estimates from multiple judges who had correlated information, but they did not look at rescaling or calibration issues.

3. The unemployment rates were downloaded from http://data.bls.gov/ $\mathrm{map} / \mathrm{servlet} / \mathrm{map}$.servlet.MapToolServlet? survey=la. To construct the statements, we first rank-ordered the states by their unemployment rates, then created all $1,250(=50 * 49 / 2)$ pairs of states, and finally indexed each pair by the difference in the rank orders of its members (cf. Wallsten, Bender, \& Li, 1999). The statements consisted of the 672 state pairs that had the greatest differences.

4. The computations of $r^{2}$ for the underconfident advisor were based on 29 (out of 30) participants. One participant had no variance among the estimates in the relevant cells. 


\section{APPENDIX}

\section{Applying the Recalibration Model to Empirical Data}

Our first goal is to obtain maximum-likelihood estimates for each participant of the scale factors $s_{j}, j=1,2,3$ corresponding to his or her use of the three advisors' estimates. The second goal is to test the constrained model in which $s_{1}=s_{2}=s_{3}=s$ against the model in which the $s_{j}$ are not restricted to be equal.

To proceed, rearrange Equation 6 to obtain

$$
\varepsilon_{j k}^{\prime}=r_{j k}^{\prime}-s_{j} p_{j k}^{\prime}
$$

We can express the error term as a deviate, $z$,

$$
z_{j k}=\frac{r_{j k}^{\prime}-s_{j} p_{j k}^{\prime}}{\sigma_{j}} .
$$

To achieve Goal 1, we establish the expression

$$
L_{\mathrm{U}}=\prod_{j k} f\left(z_{j k}\right)
$$

or its log-equivalent

$$
T_{\mathrm{U}}=\sum_{j k} \log f\left(z_{j k}\right),
$$

where $f\left(z_{j k}\right)$ refers to the likelihood of the response $r_{j k}$ (or equivalently of its log-odds deviate transformation $z_{j k}$ in Equation A1). The first goal is satisfied by finding, under suitable assumptions, the scale factors $s_{j}$ for each participant that maximize $L_{\mathrm{U}}$, the likelihood of the data, (or equivalently $T_{\mathrm{U}}$ ). The subscript $U$ denotes that the $s_{j}$ are allowed to be unequal. Computationally, it is easier to work with $T_{\mathrm{U}}$, the log-likelihood of the data. Recall from the text the three assumptions: (1) The error term $\varepsilon^{\prime}{ }_{j k}$ is identically normally distributed with mean 0 and standard deviation $\sigma_{j}$ for each advisor $j$, (2) trials are independent, and (3) the empirically derived pooled standard deviations of $r^{\prime}$ provide unbiased estimates of the $\sigma_{j}$. The quality of the estimated $s_{j}$ for each participant is assessed descriptively by comparing the predicted and observed responses, as described in the text.

Goal 2 involves testing whether the scale factors can be constrained to be equal. The model with $s_{1}=s_{2}=s_{3}$ is nested within the model in which the $s_{j}$ are not restricted to be equal (Riefer \& Batchelder, 1988). Thus, the two models can be compared using a maximum-likelihood ratio test:

$$
G^{2}=-2\left(T_{\mathrm{E}}-T_{\mathrm{U}}\right)
$$

where $T_{\mathrm{E}}$ refers to the log-likelihood function, Equation A2, for the model constrained to have equal scale factors. $G^{2}$ is asymptotically chi-squared distributed with $d f=J-1$. We used the $G^{2}$ test with $\alpha=.05$ to compare the equal and unequal scale models.

(Manuscript received January 12, 2009;

revision accepted for publication February 23, 2010.) 